\title{
Linking leptonic CP violation to quark unitarity triangle
}

\author{
Morimitsu Tanimoto and Kei Yamamoto \\ Department of Physics, Niigata University, \\ Niigata 950-2181, Japan \\ E-mail: tanimoto@muse.sc.niigata-u.ac.jp, \\ yamamoto@muse.sc.niigata-u.ac.jp
}

ABSTRACT: We study the linking between the CP violating phase of the lepton sectors and the unitarity triangle of the $B^{0}$ meson system. Antusch, King, Malinsky and Spinrath have shown that the quark mass matrices with the negligible 1-3 mixing give an interesting relation between the phase of the quark mixing matrices and CP violating measure $\phi_{2}(\alpha)$. This approach is extended by considering the $\mathrm{SO}(10)$ GUT including the Pati-Salam symmetry, which links the Yukawa matrices of the quark sector to the one of the lepton sector. We discuss the relation of the $\mathrm{CP}$ violating phases between both quark and lepton sectors as well as the mixing angles. Then, the leptonic $\mathrm{CP}$ violating phase is predicted in terms of the angle of the unitarity triangle of the $B^{0}$ meson system. The leptonic $\mathrm{CP}$ violating phase $\delta_{\mathrm{PMNS}}$ is predicted in the region $-74^{\circ} \sim-89^{\circ}$, which is the consistent with the recent T2K results. Our predicted phase is sensitive to $\phi_{2}(\alpha)$ and $\phi_{3}(\gamma)$. These predictions will be clearly tested in the future neutrino experiments as well as the Belle-II experiment.

Keywords: Neutrino Physics, CP violation, B-Physics

ARXIV EPRINT: 1501.07717 


\section{Contents}

1 Introduction $\quad 1$

2 Mixing sum rules in quarks and leptons 2

2.1 Quark mixing angle sum rules 2

$\begin{array}{ll}2.2 & \text { Lepton mixing angle sum rules }\end{array}$

3 Linking leptons to quarks $\quad 7$

$\begin{array}{lll}3.1 & \text { GUT relation between quarks and leptons } & 7\end{array}$

3.2 Predicting $\mathrm{CP}$ violating in lepton sectors 8

$\begin{array}{lll}4 & \text { Summary } & 10\end{array}$

$\begin{array}{ll}\text { A General mixing of quarks and leptons } & 11\end{array}$

\section{Introduction}

The neutrino oscillation experiments are going on the new step to reveal the CP violation in the lepton sector. The T2K experiment has confirmed the neutrino oscillation in the $\nu_{\mu} \rightarrow \nu_{e}$ appearance events [1], which provide us the new information of the CP violation of the lepton sector by combining the data of reactor experiments [2-5]. Therefore, the detailed study of the neutrino mixing including the $\mathrm{CP}$ violating phase gives us clues to reach the flavor theory [6]-[28].

On the other hand, the CP violating phase is well determined in the quark sector since the unitarity triangle of the $B^{0}$ meson system has been confirmed. The measurements of the three angles $\phi_{1}(\beta), \phi_{2}(\alpha)$ and $\phi_{3}(\gamma)$ will be improved considerably by the BelleII experiment. In the extensions of the standard model (SM), the grand unified theories (GUTs) link the Yukawa matrices of the quark sector to that of the lepton sector. Therefore, one can study the relation of the $\mathrm{CP}$ violating phases between the quark and the lepton sectors as well as the mixing angles.

The Yukawa matrices which have the 1-3 texture zeros (negligible 1-3 mixing) in both up- and down-quark sectors give us a clear relation between the Yukawa phase and the CP violating measure $\phi_{2}(\alpha)$ as discussed by Antusch, King, Malinsky and Spinrath [29]. In this paper, we extend this approach of the $\mathrm{CP}$ violating phase to the lepton sector [30,31] and discuss the link of the $\mathrm{CP}$ violating phase between the quark and lepton sectors.

By using some GUTs, the reactor angle of neutrinos is related to the quark mixing angle [32-35]. Actually, one of the authors has examined the mixing angles of both quarks and the leptons in the $\mathrm{SO}(10)$ GUT including the Pati-Salam symmetry [36, 37]. In this paper, we predict the leptonic $\mathrm{CP}$ violating phase in terms of the unitarity triangle of quarks 
by using the $\mathrm{SO}(10)$ GUT with the Pati-Salam symmetry. The leptonic CP violating phase $\delta_{\text {PMNS }}$ is predicted to be $-74^{\circ} \sim-89^{\circ}$, which is the opposite sign against the quark CP violating phase $\delta_{\mathrm{CKM}}$. This prediction is consistent with the recent T2K results [1].

The paper is organized as follows. In section 2, we present the unitarity triangle, $\phi_{1}(\beta)$, $\phi_{2}(\alpha), \phi_{3}(\gamma)$ and $J_{\mathrm{CP}}$ in terms of Yukawa phases, and examine these relations numerically by inputing the recent experimental data of the CKM matrix. The CP violating phase of the lepton sector is also discussed. In section 3, we discuss the linking between the quark $\mathrm{CP}$ violating phase and the leptonic $\mathrm{CP}$ violating phase by considering the $\mathrm{SO}(10) \mathrm{GUT}$, and predict the magnitude of the leptonic $\mathrm{CP}$ violating phase in terms of the angle of the unitarity triangle. The section 4 is devoted to the summary. In the appendix, we present the general formula of the quark and the lepton mixing.

\section{Mixing sum rules in quarks and leptons}

\subsection{Quark mixing angle sum rules}

Let us start with discussing the quark mixing angle sum rules presented by Antusch, King, Malinsky and Spinrath [29]. The quark mass matrices are given in the Yukawa sector as:

$$
\mathcal{L}_{Y}=-\bar{u}_{L}^{i}\left(M_{u}\right)_{i j} u_{R}^{j}-\overline{d_{L}^{i}}\left(M_{d}\right)_{i j} d_{R}^{j}
$$

where $M_{u}$ and $M_{d}$ are the mass matrices of the up- and the down-quarks, respectively. The mass matrices are diagonalized

$$
V_{u L}^{\dagger} M_{u} V_{u R}=\operatorname{diag}\left(m_{u}, m_{c}, m_{t}\right), \quad V_{d L}^{\dagger} M_{d} V_{d R}=\operatorname{diag}\left(m_{d}, m_{s}, m_{b}\right),
$$

where $V_{u L}, V_{u R}, V_{d L}$ and $V_{d R}$ are unitary $3 \times 3$ matrices. The CKM matrix $U_{\mathrm{CKM}}^{\prime}$ is given

$$
U_{\mathrm{CKM}}^{\prime}=V_{u L}^{\dagger} V_{d L}
$$

where the unphysical phases are included.

The unitary matrix $V_{q L}(q=u, d)$ can be written in terms of three angles $\theta_{i j}^{q}$ and three phases $\delta_{i j}^{q}$ as:

$$
V_{q L}=U_{23}^{q L} U_{13}^{q L} U_{12}^{q L}
$$

where $U_{12}^{q L}, U_{23}^{q L}$ and $U_{13}^{q L}$ are given as,

$$
U_{12}^{q L}=\left(\begin{array}{ccc}
c_{12}^{q} & s_{12}^{q} e^{-i \delta_{12}^{q}} & 0 \\
-s_{12}^{q} e^{i \delta_{12}^{q}} & c_{12}^{q} & 0 \\
0 & 0 & 1
\end{array}\right), \quad U_{23}^{q L}=\left(\begin{array}{ccc}
1 & 0 & 0 \\
0 & c_{23}^{q} & s_{23}^{q} e^{-i \delta_{23}^{q}} \\
0-s_{23}^{q} e^{i \delta_{23}^{q}} & c_{23}^{q}
\end{array}\right)
$$

and $U_{13}^{q L}$ is also analogously written. Here $c_{i j}^{q} s_{i j}^{q}$ are abbreviations for $\cos \theta_{i j}^{q}$ and $\sin \theta_{i j}^{q}$, where $\theta_{i j}^{q}$ is always made positive by the suitable choice of the phase $\delta_{i j}^{q}$. Then, we can express the CKM matrix in terms of the up- and the down-mixing matrices as:

$$
U_{\mathrm{CKM}}^{\prime}=U_{12}^{u L \dagger} U_{13}^{u L \dagger} U_{23}^{u L \dagger} U_{23}^{d L} U_{13}^{d L} U_{12}^{d L} .
$$


We can also express the CKM matrix by the products of three unitary matrices as follows:

$$
U_{\mathrm{CKM}}^{\prime}=U_{23} U_{13} U_{12},
$$

where

$$
U_{12}=\left(\begin{array}{ccc}
c_{12} & s_{12} e^{-i \delta_{12}} & 0 \\
-s_{12} e^{i \delta_{12}} & c_{12} & 0 \\
0 & 0 & 1
\end{array}\right)
$$

and so on. Three mixing angles $\theta_{i j}$ and three phases $\delta_{i j}$ appear in $U_{\mathrm{CKM}}^{\prime}$ of eq. (2.7), but two of three phases are removed away by multiplying the phase matrix in the right-hand side. On the other hand, in the PDG parametrization [38], the CKM matrix is given in terms of three mixing angles $\theta_{i j}$, which are same ones in eq. (2.8), and one phase $\delta_{\mathrm{CKM}}$ as follows:

$$
U_{\mathrm{CKM}}=\left(\begin{array}{ccc}
c_{12} c_{13} & s_{12} c_{13} & s_{13} e^{-i \delta_{\mathrm{CKM}}} \\
-s_{12} c_{23}-c_{12} s_{23} s_{13} e^{i \delta_{\mathrm{CKM}}} & c_{12} c_{23}-s_{12} s_{23} s_{13} e^{i \delta_{\mathrm{CKM}}} & s_{23} c_{13} \\
s_{12} s_{23}-c_{12} c_{23} s_{13} e^{i \delta_{\mathrm{CKM}}} & -c_{12} s_{23}-s_{12} c_{23} s_{13} e^{i \delta_{\mathrm{CKM}}} & c_{23} c_{13}
\end{array}\right),
$$

where $c_{i j}$ and $s_{i j}$ denote $\cos \theta_{i j}$ and $\sin \theta_{i j}$, respectively. Consequently, the CP violating phase $\delta_{\mathrm{CKM}}$ is expressed in terms of $\delta_{12}, \delta_{23}$ and $\delta_{13}$ as:

$$
\delta_{\mathrm{CKM}}=\delta_{13}-\delta_{23}-\delta_{12}
$$

Let us consider the phenomenological viable textures for the up- and the down-quark mass matrices, which have the 1-3 texture zeros (negligible 1-3 mixing). Therefore, taking $\theta_{13}^{d}=\theta_{13}^{u}=0$, we can express the CKM matrix

$$
U_{\mathrm{CKM}}^{\prime}=U_{12}^{u L \dagger} U_{23}^{u L \dagger} U_{23}^{d L} U_{12}^{d L}
$$

where $U_{13}^{q L}$ is the unit matrix. By using eqs. (2.7) and (2.11), we can derive the following relations,

$$
\begin{aligned}
& \theta_{23} e^{-i \delta_{23}}=\theta_{23}^{d} e^{-i \delta_{23}^{d}}-\theta_{23}^{u} e^{-i \delta_{23}^{u}}, \\
& \theta_{13} e^{-i \delta_{13}}=-\theta_{12}^{u} e^{-i \delta_{12}^{u}}\left(\theta_{23}^{d} e^{-i \delta_{23}^{d}}-\theta_{23}^{u} e^{-i \delta_{23}^{u}}\right), \\
& \theta_{12} e^{-i \delta_{12}}=\theta_{12}^{d} e^{-i \delta_{12}^{d}}-\theta_{12}^{u} e^{-i \delta_{12}^{u}},
\end{aligned}
$$

where we take the leading order in the small mixing angle by putting $c_{i j}^{u, d} \simeq 1$ and $s_{i j}^{u, d} \simeq$ $\theta_{i j}^{u, d}$. In order to see the effect of non-vanishing $\theta_{13}^{u}$ and $\theta_{13}^{u}$, we present the general formula and discussions in the appendix.

By using eqs. (2.12) and (2.13), one obtains

$$
\theta_{12}^{u}=\frac{\theta_{13}}{\theta_{23}} .
$$


By combining eqs. (2.12), (2.13) and (2.14), one gets

$$
\theta_{12}^{d} e^{-i\left(\delta_{12}^{d}-\delta_{12}\right)}=\theta_{12}-\frac{\theta_{13}}{\theta_{23}} e^{-i\left(\delta_{13}-\delta_{23}-\delta_{12}\right)},
$$

which gives

$$
\theta_{12}^{d}=\left|\theta_{12}-\frac{\theta_{13}}{\theta_{23}} e^{-i \delta_{\mathrm{CKM}}}\right|
$$

One also gets a relation by using eqs. (2.12), (2.13) and (2.14):

$$
\frac{\theta_{13} \theta_{12}}{\theta_{23}} e^{i \delta_{\mathrm{CKM}}}=-\theta_{12}^{u}\left[\theta_{12}^{d} e^{-i\left(\delta_{12}^{d}-\delta_{12}^{u}\right)}-\theta_{12}^{u}\right] .
$$

Therefore, one obtains the phase sum rule [29]

$$
\delta_{\mathrm{CKM}}=\operatorname{Arg}\left[1-\frac{\theta_{12}^{d}}{\theta_{12}^{u}} e^{-i\left(\delta_{12}^{d}-\delta_{12}^{u}\right)}\right],
$$

which is just the angle $\phi_{3}(\gamma)$ of the unitarity triangle as seen later.

The three angles of the unitarity triangle $\phi_{1}(\beta), \phi_{2}(\alpha), \phi_{3}(\gamma)$ can be expressed in terms of $\delta_{12}^{d}-\delta_{12}^{u}$ as follows:

$$
\begin{aligned}
& \phi_{1}(\beta)=\operatorname{Arg}\left[-\frac{U_{c d} U_{c b}^{*}}{U_{t d} U_{t b}^{*}}\right]=\operatorname{Arg}\left[1-\frac{\theta_{12}^{u}}{\theta_{12}^{d}} e^{-i\left(\delta_{12}^{d}-\delta_{12}^{u}\right)}\right], \\
& \phi_{2}(\alpha)=\operatorname{Arg}\left[-\frac{U_{t d} U_{t b}^{*}}{U_{u d} U_{u b}^{*}}\right]=\delta_{12}^{d}-\delta_{12}^{u}, \\
& \phi_{3}(\gamma)=\operatorname{Arg}\left[-\frac{U_{u d} U_{u b}^{*}}{U_{c d} U_{c b}^{*}}\right]=\operatorname{Arg}\left[1-\frac{\theta_{12}^{d}}{\theta_{12}^{u}} e^{-i\left(\delta_{12}^{d}-\delta_{12}^{u}\right)}\right],
\end{aligned}
$$

where the CKM matrix elements $U_{i j}$ 's are expressed in terms of $\theta_{i j}^{q}$ and $\delta_{i j}^{q}$ by using eq. (2.11). We can easily check $\phi_{1}+\phi_{2}+\phi_{3}=\pi$. It is noticed that $\phi_{3}(\gamma)$ is just $\delta_{\mathrm{CKM}}$. Thus, the $\mathrm{CP}$ violating phases are given by $\delta_{12}^{d}-\delta_{12}^{u}$ and $\theta_{12}^{d} / \theta_{12}^{u}$ in this scheme. We can also give another $\mathrm{CP}$ violating measure, the Jarlskog invariant $J_{\mathrm{CP}}$ [39], as;

$$
J_{\mathrm{CP}}=\operatorname{Im}\left[U_{u s} U_{c b} U_{u b}^{*} U_{c s}^{*}\right]=\left|U_{c b}\right|^{2} \theta_{12}^{u} \theta_{12}^{d} \sin \left(\delta_{12}^{d}-\delta_{12}^{u}\right) .
$$

Let us show numerical results in order to test the consistency of this scheme. We input the PDG data [38] for the CKM mixing elements and phase as follows:

$$
\begin{aligned}
\left|U_{u s}\right|=0.22536 \pm 0.00061, \quad\left|U_{u b}\right| & =0.00355 \pm 0.00015, \quad\left|U_{c b}\right|=0.0414 \pm 0.0012 \\
\delta_{\mathrm{CKM}} & =\phi_{3}(\gamma)=68.0_{-8.5^{\circ}}^{+8.0^{\circ}}
\end{aligned}
$$

Then we can obtain $\delta_{12}^{d}-\delta_{12}^{u}$ and $\theta_{12}^{d} / \theta_{12}^{u}$ by using eqs. (2.15), (2.17) and (2.19), and so we can calculate $\sin 2 \phi_{1}(\beta), \phi_{2}(\alpha)$ and $J_{\mathrm{CP}}$ by using eqs. (2.20) and (2.21).

Taking account of the experimental error with $90 \%$ C.L. for the input data, we plot the calculated regions of $\sin 2 \phi_{1}(\beta), \phi_{2}(\alpha)$ and $J_{\mathrm{CP}}$ on the planes of $\sin 2 \phi_{1}(\beta)-\phi_{2}(\alpha)$, 


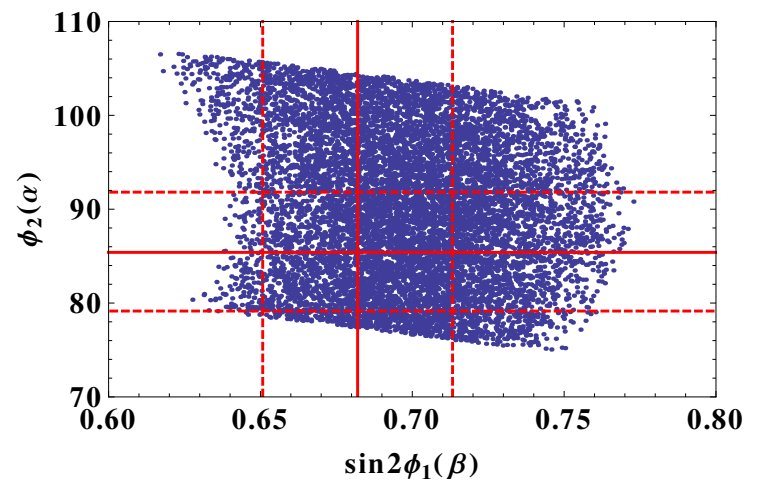

Figure 1. The predicted region on the $\sin 2 \phi_{1}(\beta)-\phi_{2}(\alpha)$ plane. The solid and dashed lines denote the central values and the errors with $90 \%$ C.L. of the experimental data, respectively.

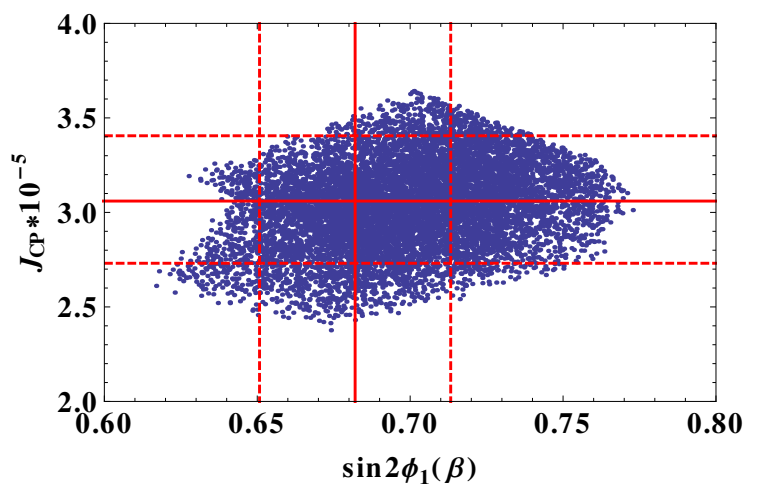

Figure 2. The predicted region on the $\sin 2 \phi_{1}(\beta)-J_{\mathrm{CP}}$ plane. The solid and dashed lines denote the central values and the errors with $90 \%$ C.L. of the experimental data, respectively.

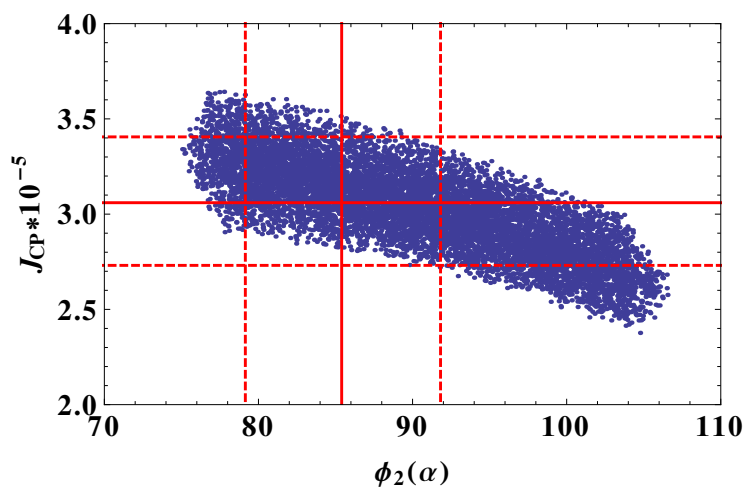

Figure 3. The predicted region on the $\phi_{2}(\alpha)-J_{\mathrm{CP}}$ plane. The solid and dashed lines are same in figures 1 and 2 .

$\sin 2 \phi_{1}(\beta)-J_{\mathrm{CP}}$ and $\phi_{2}(\alpha)-J_{\mathrm{CP}}$ in figures 1,2 and 3 , respectively. In these figures, the following experimental bounds with $90 \%$ C.L. are also shown:

$$
\sin 2 \phi_{1}(\beta)=0.682 \pm 0.019, \quad \phi_{2}(\alpha)=85.4_{-3.8^{\circ}}^{\circ+3 . \circ^{\circ}}, \quad J_{\mathrm{CP}}=3.06_{-0.20}^{+0.21} \times 10^{-5} .
$$

The calculated $\sin 2 \phi_{1}(\beta), \phi_{2}(\alpha)$ and $J_{\mathrm{CP}}$ are consistent with the experimental data although some regions are excluded by the experimental bounds. Thus, the $\mathrm{CP}$ violation is 


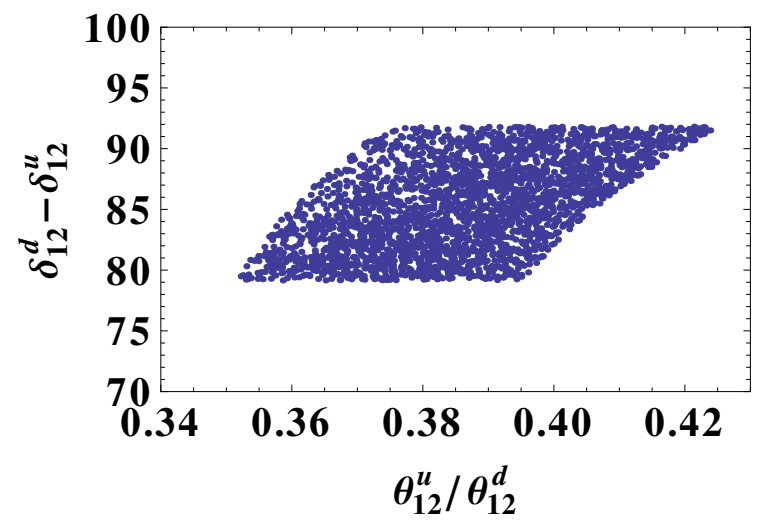

Figure 4. The allowed region of $\delta_{12}^{d}-\delta_{12}^{u}$ and $\theta_{12}^{d} / \theta_{12}^{u}$ with the constraint of the unitarity triangle.

successfully expressed in terms of $\delta_{12}^{d}-\delta_{12}^{u}$ and $\theta_{12}^{d} / \theta_{12}^{u}$, which come from the quark mass matrices. By imposing constraints of the experimental data of the unitarity triangle in eq. (2.23), we finally obtain the allowed region of $\delta_{12}^{d}-\delta_{12}^{u}$ and $\theta_{12}^{d} / \theta_{12}^{u}$ as shown in figure 4 . Taking this allowed region of $\delta_{12}^{d}-\delta_{12}^{u}$, we will discuss the CP violating phase in the lepton sector.

\subsection{Lepton mixing angle sum rules}

Similar discussions lead to sum rules for the mixing angles and the CP violating phase in the lepton sector [30, 31]. For the lepton mixing [40, 41], we write a general unitarity matrix $U_{\mathrm{PMNS}}^{\prime}$ including the Majorana phases $\varphi_{1}, \varphi_{2}$ and the Dirac phase $\delta_{\mathrm{PMNS}}$ as follows:

$$
U_{\mathrm{PMNS}}^{\prime}=\operatorname{diag}\left(e^{i \delta_{e}}, e^{i \delta_{\mu}}, e^{i \delta_{\tau}}\right) \cdot U_{\mathrm{PMNS}} \cdot \operatorname{diag}\left(e^{-i \varphi_{1} / 2}, e^{-i \varphi_{2} / 2}, 1\right) .
$$

Here $U_{\text {PMNS }}$ is the lepton mixing matrix corresponding to the quark one of eq. (2.9), in which $\theta_{i j}$ and $\delta_{i j}$ are redefined in the lepton sector. We replace the quark mixing angles and the phases $\theta_{i j}^{d}, \theta_{i j}^{u}, \delta_{i j}^{d}, \delta_{i j}^{u}$ with the lepton ones $\theta_{i j}^{e}, \theta_{i j}^{\nu}, \delta_{i j}^{e}, \delta_{i j}^{\nu}$, where superscripts $e$ and $\nu$ correspond to the charged lepton and the neutrino, respectively.

The observed mixing angles $\theta_{23}$ and $\theta_{12}$ are order one while $\theta_{13}$ is order of the Cabibbo angle in the experimental data. Therefore, it is reasonable to suppose $\theta_{13}^{e}=\theta_{13}^{\nu}=0$ as well as in the quark sector. So, we can take the PNMS matrix to be

$$
U_{\mathrm{PMNS}}^{\prime}=U_{12}^{e L \dagger} U_{23}^{e L \dagger} U_{23}^{\nu L} U_{12}^{\nu L} .
$$

Then, the PMNS mixing angles $\theta_{i j}$ 's are given in terms of $\theta_{i j}^{e}, \theta_{i j}^{\nu}$, and $\delta_{i j}^{e}, \delta_{i j}^{\nu}$ as follows:

$$
\begin{aligned}
c_{13} s_{23} e^{-i \delta_{23}} & =s_{23}^{\nu} e^{-i \delta_{23}^{\nu}}-\theta_{23}^{e} c_{23}^{\nu} e^{-i \delta_{23}^{e}} \\
\theta_{13} e^{-i \delta_{13}} & =-\theta_{12}^{e} e^{-i \delta_{12}^{e}}\left(s_{23}^{\nu} e^{-i \delta_{23}^{\nu}}-\theta_{23}^{e} c_{23}^{\nu} e^{-i \delta_{23}^{e}}\right), \\
c_{13} s_{12} e^{-i \delta_{12}} & =s_{12}^{\nu} e^{-i \delta_{12}^{\nu}}-\theta_{12}^{e} c_{23}^{\nu} c_{12}^{\nu} e^{-i \delta_{12}^{e}}
\end{aligned}
$$

where we take $\cos \theta_{i j}^{e} \simeq 1, \sin \theta_{i j}^{e} \simeq \theta_{i j}^{e}$ and $\sin \theta_{13} \simeq \theta_{13}$. The leptonic CP phases can be extracted via

$$
\delta_{12}=\frac{1}{2}\left(\varphi_{2}-\varphi_{1}\right), \quad \delta_{23}=-\frac{1}{2} \varphi_{2}, \quad \delta_{13}=\delta_{\mathrm{PMNS}}-\frac{1}{2} \varphi_{1} .
$$


In this situation, the neutrino large mixing angles come from the neutrino mass matrix, and the reactor angle $\theta_{13}$ is given by the correction from the charged lepton sector. By eqs. (2.26) and (2.27), we obtain

$$
\theta_{13} e^{-i \delta_{13}}=-\theta_{12}^{e} c_{13} s_{23} e^{-i\left(\delta_{12}^{e}+\delta_{23}\right)},
$$

which gives us simple sum rules for mixing angles and phases

$$
\theta_{13}=\theta_{12}^{e} s_{23}, \quad \delta_{13}=\delta_{12}^{e}+\delta_{23}-\pi
$$

Supposing $\theta_{23}^{e} \ll 0.1$ in eq. (2.26), we obtain

$$
s_{23} \simeq s_{23}^{\nu}, \quad \delta_{23}=\delta_{23}^{\nu} .
$$

On the other hand, eq. (2.28) turns to

$$
c_{13} s_{12} e^{-i \delta_{12}}=-\theta_{12}^{e} c_{23}^{\nu} c_{12}^{\nu} e^{-i \delta_{12}^{e}}\left(1-\frac{s_{12}^{\nu}}{\theta_{12}^{e} c_{23}^{\nu} c_{12}^{\nu}} e^{-i\left(\delta_{12}^{\nu}-\delta_{12}^{e}\right)}\right),
$$

which leads to

$$
s_{12}=\left|s_{12}^{\nu} e^{-i\left(\delta_{12}^{\nu}-\delta_{12}^{e}\right)}-\theta_{12}^{e} c_{23}^{\nu} c_{12}^{\nu}\right|, \quad \delta_{12}=\delta_{12}^{e}-\phi-\pi,
$$

where $\phi$ is given by

$$
\phi=\operatorname{Arg}\left[1-\frac{s_{12}^{\nu}}{\theta_{12}^{e} c_{23}^{\nu} c_{12}^{\nu}} e^{-i\left(\delta_{12}^{\nu}-\delta_{12}^{e}\right)}\right] .
$$

Therefore, the leptonic $\mathrm{CP}$ violating phase $\delta_{\mathrm{PMNS}}$ is given as

$$
\delta_{\mathrm{PMNS}}=\delta_{13}-\delta_{23}-\delta_{12}=\phi-2 \pi \equiv \phi .
$$

Thus, the leptonic CP violating phase is given by $\delta_{12}^{e}-\delta_{12}^{\nu}$, which is similar to $\delta_{\mathrm{CKM}}$ of the quark sector in eq. (2.19). It is remarked that $\delta_{\mathrm{PMNS}}$ is given by the replacement of $d \rightarrow \nu$ and $u \rightarrow e$ in $\delta_{\mathrm{CKM}}$. This situation is understandable since the CKM matrix is $V_{u L}^{\dagger} V_{d L}$ while the PMNS matrix is $V_{e L}^{\dagger} V_{\nu L}$.

\section{Linking leptons to quarks}

\subsection{GUT relation between quarks and leptons}

The GUT models relate $\theta_{12}^{e}$ to the quark mixing angle. Especially, $\theta_{12}^{e} \simeq \theta_{C}$ with $\theta_{C}$ being the Cabibbo angle, comes from the GUT relations between the down-type Yukawa matrix $Y_{d}$ and the charged lepton Yukawa matrix $Y_{e}$, and then one obtains the successful relation $\theta_{13} \simeq \theta_{C} / \sqrt{2}$ from eq. (2.31) [30-33].

In order to link the lepton mass matrices to the quark mass matrices, we consider the $\mathrm{SO}(10)$ GUT, which is broken down to the SM gauge group through the Pati-Salam symmetry [36, 37]. In this setup, the quark Yukawa matrices $Y_{d}, Y_{u}$, the charged lepton Yukawa matrix $Y_{e}$ and the Dirac neutrino Yukawa matrix $Y_{\nu}$ are symmetric ones, and 
$Y_{d}=Y_{e}$ and $Y_{u}=Y_{\nu}$ are guaranteed except for the group theoretical Clebsch-Gordan (CG) factors in each element, which depends on the GUT operators. The left-handed Majorana neutrino mass matrix is not directly obtained from the up-quark Yukawa matrix $Y_{u}$ since it is related with the Dirac neutrino mass matrix with the seesaw mechanism, and we therefore have some freedom coming from the right-handed neutrino mass matrix $M_{R}$. The remarkable difference of the mixing angles between the CKM matrix and the PMNS matrix is due to the flavor structure in the right-handed neutrino mass matrix $M_{R}$.

Now, one can take $[32,33]$

$$
\theta_{12}^{e}=\theta_{12}^{d},
$$

which leads to $\theta_{13} \simeq \theta_{C} / \sqrt{2}$. This relation was examined by the renormalization group running [30], and then, it is justified to be a good approximation at low energies. Therefore, we use it as the low energy relation in our work.

As well as the mixing angles, the phases of the Yukawa matrix elements are also related with each other in both quark and lepton sectors. Since $Y_{d}=Y_{e}$ and $Y_{u}=Y_{\nu}$ except for CG factors, we can discuss the relation of the Dirac phases between the quark and the lepton sectors. In order to simplify the discussion, we take the real base for $Y_{u}$ and $Y_{\nu}$, that is $\delta_{12}^{u}=\delta_{12}^{\nu}=0$ and $\delta_{23}^{u}=\delta_{23}^{\nu}=0$. One can take this base in general as follows. Through multiplying both side of eq. (2.12), eq. (2.13) and eq. (2.14) by the phase factors $e^{i \delta_{23}^{u}}$, $e^{i\left(\delta_{12}^{u}+\delta_{23}^{u}\right)}$ and $e^{i \delta_{12}^{u}}$, respectively, the phases $e^{i \delta_{12}^{u}}$ and $e^{i \delta_{23}^{u}}$ are removed in the up-quark mixing, while the down-quark phases turn to $\delta_{i j}^{d}-\delta_{i j}^{u}$ as seen in the right-hand sides. The phases of the left-hand sides can be again redefined as $\delta_{i j}$. In the lepton sector, the same discussion is available in eqs. (2.26), (2.27) and (2.28).

Due to $Y_{d}=Y_{e}$ with the real $Y_{u}$ and $Y_{\nu}$, we obtain

$$
\delta_{12}^{e}=\delta_{12}^{d} .
$$

The neutrino flavor mixing is much different from the up-quark flavor mixing because of the seesaw mechanism. If the right-handed neutrino mass matrix $M_{R}$ is real apart from the Majorana phases in this base, Dirac phases do not appear in the neutrino mixing matrix. The CP violating Dirac phases appear only in the charged lepton sector. A typical example is the tri-bimaximal mixing, which is the real mixing with $\theta_{13}^{\nu}=0[42,43]$. Then, the leptonic $\mathrm{CP}$ violating phase comes from the charged lepton sector as well as the nonvanishing $\theta_{13}^{\nu}$.

Taking account of the phase redefinition, we rewrite eq. (3.2) as

$$
\delta_{12}^{e}-\delta_{12}^{\nu}=\delta_{12}^{d}-\delta_{12}^{u},
$$

which is the link of the $\mathrm{CP}$ violating phase between quarks and leptons. By using this relation, we predict the $\mathrm{CP}$ violating phase of leptons, $\delta_{\mathrm{PMNS}}$.

\subsection{Predicting CP violating in lepton sectors}

Let us consider the case, in which the mixing angles and the phases of the quark and lepton Yukawa matrices satisfy the relations in eqs. (3.1) and (3.3), respectively. Then, the $\delta_{\mathrm{PMNS}}$ 


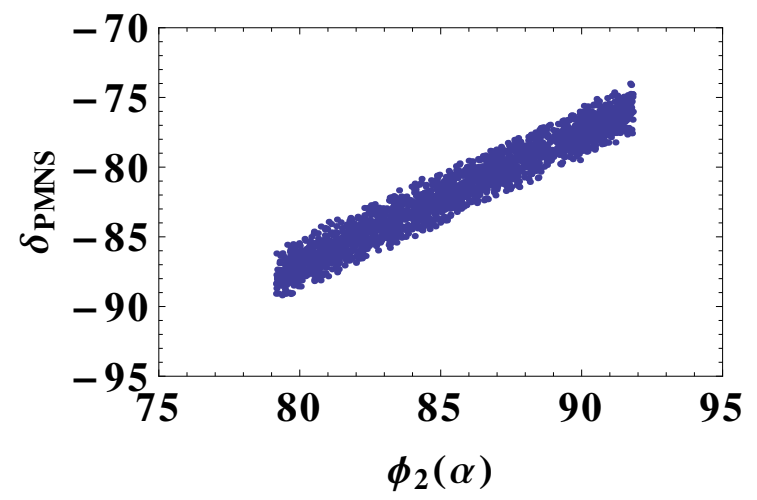

Figure 5. Predicted $\delta_{\mathrm{PMNS}}$ vs. $\phi_{2}(\alpha)$.

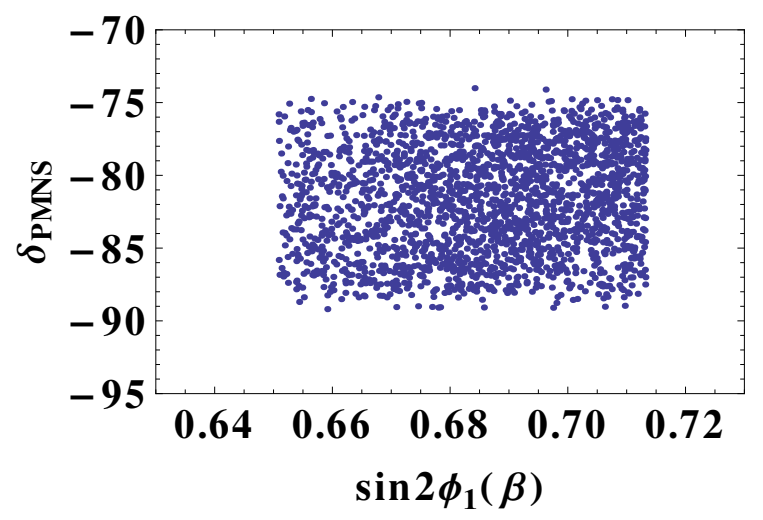

Figure 6. Predicted $\delta_{\mathrm{PMNS}}$ vs. $\sin 2 \phi_{1}(\beta)$.

is given in terms of $\phi_{2}(\alpha)$ by using eq. (2.36):

$$
\delta_{\mathrm{PMNS}}=\phi=\operatorname{Arg}\left[1-\frac{s_{12}^{\nu}}{\theta_{12}^{e} c_{23}^{\nu} c_{12}^{\nu}} e^{i \phi_{2}(\alpha)}\right] .
$$

We can calculate $\delta_{\text {PMNS }}$ without assuming the flavor structure of the neutrino sector since the values of $\theta_{23}^{\nu}$ and $\theta_{12}^{\nu}$ are obtained by the experimental data as seen in eqs. (2.32) and (2.34), while $\theta_{12}^{e}$ is fixed by eq. (3.1). For input data in our calculations, we use the results of the global analysis of the neutrino oscillation experiments for $\theta_{12}, \theta_{23}$ and $\theta_{13}[44-46]$.

Now we predict $\delta_{\text {PMNS }}$ in terms of the $\mathrm{CP}$ violating phase of the quarks by using eq. (3.4). We obtain $\delta_{\mathrm{PMNS}}$ in the region of $-74^{\circ} \sim-89^{\circ}$ which is consistent with the recent T2K results with $90 \%$ C.L. [1]; $-1.18 \pi<\delta_{\mathrm{CP}}<0.15 \pi\left(-0.91 \pi<\delta_{\mathrm{CP}}<-0.08 \pi\right)$ for the normal (inverted) hierarchy of neutrinos. The predict $\delta_{\mathrm{PMNS}}$ is the opposite sign against $\delta_{\mathrm{CKM}}$, which is due to that $\delta_{\mathrm{PMNS}}$ is given by replacement of $d \rightarrow \nu$ and $u \rightarrow e$ in $\delta_{\mathrm{CKM}}$.

We show the predicted $\delta_{\mathrm{PMNS}}$ versus $\phi_{2}(\alpha), \sin 2 \phi_{1}(\beta), \phi_{3}(\gamma)$ and $J_{\mathrm{CP}}$ in figures $5,6,7$ and 8 , respectively. As seen in figure $5, \delta_{\mathrm{PMNS}}$ has a linear dependence of $\phi_{2}(\alpha)$ with somewhat uncertainty, which is due to the error of input data, $\theta_{12}$ and $\theta_{23}$, as seen in eqs. (2.32), (2.34) and (3.4). The more precise data of $\phi_{2}(\alpha)$ in the $B^{0}$ meson system gives 


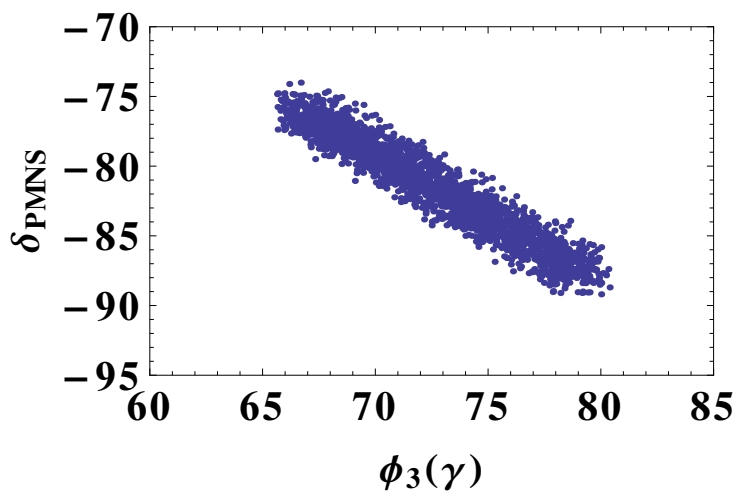

Figure 7. Predicted $\delta_{\text {PMNS }}$ vs. $\phi_{3}(\gamma)$.

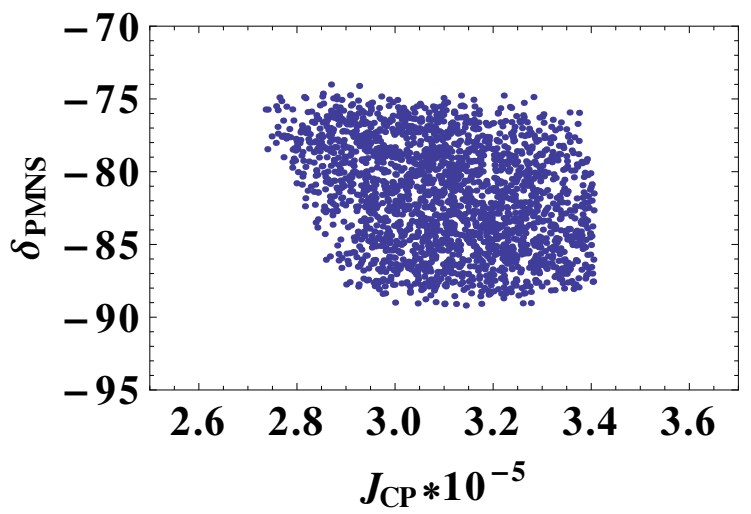

Figure 8. Predicted $\delta_{\mathrm{PMNS}}$ vs. $J_{\mathrm{CP}}$.

the more accurate prediction of $\delta_{\mathrm{PMNS}}$. If the $\phi_{2}(\alpha)$ is just a right angle, $\delta_{\mathrm{PMNS}}$ should be $-75^{\circ} \sim-80^{\circ}$.

On the other hand, $\delta_{\mathrm{PMNS}}$ is insensitive to $\sin 2 \phi_{1}(\beta)$. As seen in eq. (2.20), $\sin 2 \phi_{1}(\beta)$ depends on $\theta_{12}^{u} / \theta_{12}^{d}$, where the uncertainty of $\theta_{12}^{d}$ and $\theta_{12}^{u}$ comes from the experimental errorbar of the CKM matrix elements $\left|U_{u b}\right|,\left|U_{c b}\right|$ and $\phi_{3}(\gamma)$ as seen in eq. (2.17). Therefore, the $\sin 2 \phi_{1}(\beta)$ dependence of $\delta_{\text {PMNS }}$ will be seen after the more precise determination of the CKM matrix elements $\left|U_{u b}\right|,\left|U_{c b}\right|$ and $\phi_{3}(\gamma)$ in the future experiments.

It is found that $\delta_{\text {PMNS }}$ is sensitive to $\phi_{3}(\gamma)$, which also depends on $\theta_{12}^{d} / \theta_{12}^{u}$. In this case, since $\theta_{12}^{d}$ is given for the fixed $\phi_{3}(\gamma)$, the uncertainty of $\theta_{12}^{d}$ is significantly reduced. The predicted $\delta_{\mathrm{PMNS}}$ is insensitive to $J_{\mathrm{CP}}$.

\section{Summary}

We have discussed the linking between the $\mathrm{CP}$ violating phase of the lepton sector and the unitarity triangle of the quark sector. The 1-3 texture zeros (negligible 1-3 mixing) in the up- and the down-quark mass matrices have given us an interesting relation between the Yukawa phase and $\mathrm{CP}$ violating measure $\phi_{2}(\alpha)[29]$. We have examined this relation by using the recent data of three angles of the unitarity triangle in the $B^{0}$ meson system and the $\mathrm{CP}$ violating measure $J_{\mathrm{CP}}$. We have found that the $\mathrm{CP}$ violation is successfully expressed in terms of $\delta_{12}^{d}-\delta_{12}^{u}$ and $\theta_{12}^{d} / \theta_{12}^{u}$, which come from the quark mass matrices. 
We have extended this approach by considering the $\mathrm{SO}(10)$ GUT including the PatiSalam symmetry, which links the Yukawa couplings of the quark sector to the one of the lepton sector. Then, there is a simple relation of the CP violating Dirac phases between both quark and lepton sectors as well as the mixing angles. We have predicted the leptonic $\mathrm{CP}$ violating phase in terms of the angle of the unitaity triangle of quarks. The leptonic $\mathrm{CP}$ violating phase $\delta_{\mathrm{PMNS}}$ is in the region $-74^{\circ} \sim-89^{\circ}$, which is the opposite sign against $\delta_{\mathrm{CKM}}$. The predicted value is the consistent with the recent T2K results. Our predicted $\delta_{\text {PMNS }}$ is sensitive to $\phi_{2}(\alpha)$ and $\phi_{3}(\gamma)$, on the other hand, insensitive to $\sin 2 \phi_{1}(\beta)$ and $J_{\mathrm{CP}}$. Our predictions will be tested in the future neutrino experiments and the BelleII experiment.

\section{Acknowledgments}

M.T.and K.Y are supported by JSPS Grand-in-Aid for Scientific Research, No.24654062. and No.25-5222, respectively. M.T would like to thank Sin Kyu Kang for helpful discussion.

\section{A General mixing of quarks and leptons}

The CKM matrix is given in general as:

$$
U_{\mathrm{CKM}}^{\prime}=U_{12}^{u L \dagger} U_{13}^{u L \dagger} U_{23}^{u L \dagger} U_{23}^{d L} U_{13}^{d L} U_{12}^{d L}
$$

Then, we have mixing sum rules:

$$
\begin{aligned}
& \theta_{23} e^{-i \delta_{23}}=\left(s_{23}^{d} e^{-i \delta_{23}^{d}}-s_{23}^{u} e^{-i \delta_{23}^{u}}\right)+s_{12}^{u} e^{i \delta_{12}^{u}}\left(s_{13}^{d} e^{-i \delta_{13}^{d}}-s_{13}^{u} e^{-i \delta_{13}^{u}}\right) \\
& \theta_{13} e^{-i \delta_{13}}=-s_{12}^{u} e^{-i \delta_{12}^{u}}\left(s_{23}^{d} e^{-i \delta_{23}^{d}}-s_{23}^{u} e^{-i \delta_{23}^{u}}\right)+\left(s_{13}^{d} e^{-i \delta_{13}^{d}}-s_{13}^{u} e^{-i \delta_{13}^{u}}\right), \\
& \theta_{12} e^{-i \delta_{12}}=\left(s_{12}^{d} e^{-i \delta_{12}^{d}}-s_{12}^{u} e^{-i \delta_{12}^{u}}\right)+s_{13}^{u} e^{-i \delta_{13}^{u}}\left(s_{23}^{d} e^{-i \delta_{23}^{d}}-s_{23}^{u} e^{-i \delta_{23}^{u}}\right)
\end{aligned}
$$

where we keep the second order of the small mixing angles by putting $c_{i j}^{u, d} \simeq 1$ and $s_{i j}^{u, d} \ll 1$.

The angle of the CP violation $\phi_{2}(\alpha)$ is defined in terms of the CKM matrix elements as

$$
\phi_{2}(\alpha)=\operatorname{Arg}\left[-\frac{U_{t d} U_{t b}^{*}}{U_{u d} U_{u b}^{*}}\right] \simeq \operatorname{Arg}\left[-\frac{U_{t d}}{U_{u b}^{*}}\right]
$$

where $U_{u b}$ is given by $\theta_{13} e^{-i \delta_{13}}$ in eq. (A.2). On the other hand, $U_{t d}$ is expressed as

$$
U_{t d}=s_{12}^{d} e^{i \delta_{12}^{d}}\left(s_{23}^{d} e^{i \delta_{23}^{d}}-s_{23}^{u} e^{i \delta_{23}^{u}}\right)+\left(s_{13}^{u} e^{i \delta_{13}^{u}}-s_{13}^{d} e^{i \delta_{13}^{d}}\right) .
$$

Then, $\phi_{2}(\alpha)$ is obtained as follows:

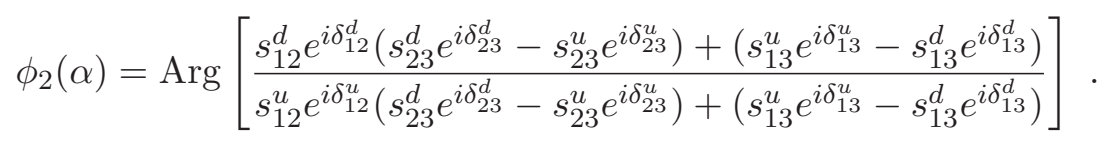

We obtain easily $\phi_{2}(\alpha)=\delta_{12}^{d}-\delta_{12}^{u}$ if $s_{13}^{u}=s_{13}^{d}=0$ is put. In the case of non-vanishing $s_{13}^{u}$ and $s_{13}^{d}, \phi_{2}(\alpha)$ depends on the extra parameters of the (1-3) mixing in additon to $\delta_{12}^{d}-\delta_{12}^{u}$. 
Even if $s_{13}^{d}$ has a non-vanishing value with $s_{13}^{u}=0$, this situation is not changed, and vice versa. Thus, we lose the predictive power for the CP violating phase unless $s_{13}^{u}=s_{13}^{d}=0$.

Instead of $s_{13}^{u}=s_{13}^{d}=0$, if we put the $s_{12}^{u}=s_{12}^{d}=0$ or $s_{23}^{u}=s_{23}^{d}=0$ we get $\phi_{2}(\alpha)=0$ as seen in eq. (A.5), which is inconsistent with the experimental data of the CKM mixing. In conclusion, $s_{13}^{u}=s_{13}^{d}=0$ is the minimal choice in the standpoint of the texture zeros to discuss the $\mathrm{CP}$ violation of the CKM mixing.

Let us discuss the PMNS matrix, which is given as,

$$
U_{\mathrm{PMNS}}^{\prime}=U_{12}^{e L \dagger} U_{13}^{e L \dagger} U_{23}^{e L \dagger} U_{23}^{\nu L} U_{13}^{\nu L} U_{12}^{\nu L} .
$$

Taking $s_{i j}^{e} \ll 1$ in the charged leptons and the mixing angles with order one in the neutrinos, we obtain

$$
\begin{aligned}
c_{13} s_{23} e^{-i \delta_{23}} & =c_{13}^{\nu} s_{23}^{\nu} e^{-i \delta_{23}^{\nu}}-s_{23}^{e} c_{23}^{\nu} c_{13}^{\nu} e^{-i \delta_{23}^{e}}+s_{12}^{e} s_{13}^{\nu} e^{-i\left(\delta_{13}^{e}-\delta_{12}^{e}\right)}, \\
s_{13} e^{-i \delta_{13}} & =-s_{12}^{e} c_{13}^{\nu} e^{-i \delta_{12}^{e}}\left(s_{23}^{\nu} e^{-i \delta_{23}^{\nu}}-s_{23}^{e} c_{23}^{\nu} e^{-i \delta_{23}^{e}}\right)+s_{13}^{\nu} e^{-i \delta_{13}^{\nu}}-s_{13}^{e} c_{13}^{\nu} c_{23}^{\nu} e^{-i \delta_{13}^{e}}, \\
c_{13} s_{12} e^{-i \delta_{12}} & =c_{13}^{\nu} s_{12}^{\nu} e^{-i \delta_{12}^{\nu}}-s_{12}^{e} c_{12}^{\nu} c_{23}^{\nu} e^{-i \delta_{12}^{e}}+s_{13}^{e} s_{23}^{\nu} c_{12}^{\nu} e^{-i\left(\delta_{13}^{e}-\delta_{23}^{\nu}\right)} .
\end{aligned}
$$

Since the observed $s_{23}$ and $s_{12}$ are large, we cannot take $s_{23}^{\nu}=0$ and $s_{12}^{\nu}=0$ if the charged lepton mixing angles is assumed to be order of the quark ones through the GUT model. Therefore, the input of $s_{13}^{\nu}=0$ in addition to $s_{13}^{e}=0$ is a reasonable one. If we take $s_{13}^{\nu} \neq 0$, it is an extra parameter as seen in eq. (A.7). Then, we lose the predictive power for $\theta_{13}$ and the $\mathrm{CP}$ violating phase $\delta_{\mathrm{PMNS}}$.

Open Access. This article is distributed under the terms of the Creative Commons Attribution License (CC-BY 4.0), which permits any use, distribution and reproduction in any medium, provided the original author(s) and source are credited.

\section{References}

[1] T2K collaboration, K. Abe et al., Observation of electron neutrino appearance in a muon neutrino beam, Phys. Rev. Lett. 112 (2014) 061802 [arXiv:1311.4750] [INSPIRE].

[2] Daya BAY collaboration, F.P. An et al., Observation of electron-antineutrino disappearance at Daya Bay, Phys. Rev. Lett. 108 (2012) 171803 [arXiv:1203.1669] [InSPIRE].

[3] RENO collaboration, J.K. Ahn et al., Observation of Reactor Electron Antineutrino Disappearance in the RENO experiment, Phys. Rev. Lett. 108 (2012) 191802 [arXiv: 1204.0626] [INSPIRE].

[4] MINOS collaboration, P. Adamson et al., Measurement of neutrino and antineutrino oscillations using beam and atmospheric data in MINOS, Phys. Rev. Lett. 110 (2013) 251801 [arXiv: 1304.6335] [INSPIRE].

[5] Double CHOOZ collaboration, Y. Abe et al., Background-independent measurement of $\theta_{13}$ in Double CHOOZ, Phys. Lett. B 735 (2014) 51 [arXiv:1401.5981] [INSPIRE].

[6] S.K. Kang, C.S. Kim and J.D. Kim, Neutrino masses and leptonic CP-violation, Phys. Rev. D 62 (2000) 073011 [hep-ph/0004020] [InSPIRE].

[7] M. Fukugita and M. Tanimoto, Lepton flavor mixing matrix and CP-violation from neutrino oscillation experiments, Phys. Lett. B 515 (2001) 30 [hep-ph/0107082] [INSPIRE]. 
[8] C. Giunti and M. Tanimoto, CP violation in bilarge lepton mixing, Phys. Rev. D 66 (2002) 113006 [hep-ph/0209169] [INSPIRE].

[9] Z.-z. Xing, Nearly tri bimaximal neutrino mixing and CP-violation, Phys. Lett. B 533 (2002) 85 [hep-ph/0204049] [INSPIRE].

[10] B. Adhikary and A. Ghosal, Constraining it CP-violation in a softly broken $A_{4}$ symmetric Model, Phys. Rev. D 75 (2007) 073020 [hep-ph/0609193] [inSPIRE].

[11] B. Adhikary and A. Ghosal, Nonzero $U_{e 3}, C P$-violation and leptogenesis in a see-saw type softly broken $A_{4}$ symmetric model, Phys. Rev. D 78 (2008) 073007 [arXiv:0803.3582] [INSPIRE].

[12] G.C. Branco, R.G. Felipe and F.R. Joaquim, Leptonic CP-violation, Rev. Mod. Phys. 84 (2012) 515 [arXiv:1111.5332] [InSPIRE].

[13] G.C. Branco, R. Gonzalez Felipe, F.R. Joaquim and H. Serodio, Spontaneous leptonic CP-violation and nonzero $\theta_{13}$, Phys. Rev. D 86 (2012) 076008 [arXiv: 1203.2646] [InSPIRE].

[14] Y.H. Ahn and S.K. Kang, Non-zero $\theta_{13}$ and CP-violation in a model with $A_{4}$ flavor symmetry, Phys. Rev. D 86 (2012) 093003 [arXiv:1203.4185] [INSPIRE].

[15] H. Ishimori, S. Khalil and E. Ma, CP phases of neutrino mixing in a supersymmetric B-L Gauge Model with $T_{7}$ lepton flavor symmetry, Phys. Rev. D 86 (2012) 013008 [arXiv: 1204.2705] [INSPIRE].

[16] H. Ishimori and E. Ma, New simple $A_{4}$ neutrino model for nonzero $\theta_{13}$ and large $\delta_{C P}$, Phys. Rev. D 86 (2012) 045030 [arXiv: 1205.0075] [INSPIRE].

[17] W. Rodejohann and H. Zhang, Simple two parameter description of lepton mixing, Phys. Rev. D 86 (2012) 093008 [arXiv:1207.1225] [INSPIRE].

[18] D. Marzocca, S.T. Petcov, A. Romanino and M.C. Sevilla, Nonzero $\left|U_{e 3}\right|$ from charged lepton corrections and the atmospheric neutrino mixing angle, JHEP 05 (2013) 073 [arXiv: 1302.0423] [INSPIRE].

[19] P. Ballett, S.F. King, C. Luhn, S. Pascoli and M.A. Schmidt, Testing atmospheric mixing sum rules at precision neutrino facilities, Phys. Rev. D 89 (2014) 016016 [arXiv: 1308.4314] [INSPIRE].

[20] P. Ballett, S.F. King, C. Luhn, S. Pascoli and M.A. Schmidt, Testing solar lepton mixing sum rules in neutrino oscillation experiments, JHEP 12 (2014) 122 [arXiv:1410.7573] [INSPIRE].

[21] S.F. King, A. Merle, S. Morisi, Y. Shimizu and M. Tanimoto, Neutrino mass and mixing: from theory to experiment, New J. Phys. 16 (2014) 045018 [arXiv:1402.4271] [INSPIRE].

[22] Z.-z. Xing and S. Zhou, A partial $\mu-\tau$ symmetry and its prediction for leptonic CP-violation, Phys. Lett. B 737 (2014) 196 [arXiv:1404.7021] [INSPIRE].

[23] G.C. Branco, M.N. Rebelo, J.I. Silva-Marcos and D. Wegman, Quasidegeneracy of Majorana neutrinos and the origin of large leptonic mixing, Phys. Rev. D 91 (2015) 013001 [arXiv: 1405.5120] [INSPIRE].

[24] S.T. Petcov, Predicting the values of the leptonic CP-violation phases in theories with discrete flavour symmetries, Nucl. Phys. B 892 (2015) 400 [arXiv:1405.6006] [INSPIRE].

[25] S.K. Kang and C.S. Kim, Prediction of leptonic CP phase from perturbatively modified tribimaximal (or bimaximal) mixing, Phys. Rev. D 90 (2014) 077301 [arXiv:1406.5014] [INSPIRE].

[26] Y. Shimizu, M. Tanimoto and K. Yamamoto, Predicting CP-violation in deviation from tri-bimaximal mixing of neutrinos, Mod. Phys. Lett. A 30 (2015) 1550002 [arXiv: 1405.1521] [INSPIRE]. 
[27] I. Girardi, S.T. Petcov and A.V. Titov, Determining the Dirac CP-violation phase in the neutrino mixing matrix from sum rules, arXiv:1410.8056 [INSPIRE].

[28] S.K. Kang and M. Tanimoto, Prediction of leptonic CP phase in $A_{4}$ symmetric model, arXiv: 1501.07428 [INSPIRE].

[29] S. Antusch, S.F. King, M. Malinsky and M. Spinrath, Quark mixing sum rules and the right unitarity triangle, Phys. Rev. D 81 (2010) 033008 [arXiv:0910.5127] [INSPIRE].

[30] S. Antusch, C. Gross, V. Maurer and C. Sluka, $\theta_{13}^{P M N S}=\theta_{C} / \sqrt{2}$ from GUTs, Nucl. Phys. B 866 (2013) 255 [arXiv: 1205.1051] [INSPIRE].

[31] S. Antusch, C. Gross, V. Maurer and C. Sluka, A flavour GUT model with $\theta_{13}^{P M N S} \simeq \theta_{C} / \sqrt{2}$, Nucl. Phys. B 877 (2013) 772 [arXiv:1305.6612] [inSPIRE].

[32] S. Antusch and V. Maurer, Large neutrino mixing angle $\theta_{13}^{M N S}$ and quark-lepton mass ratios in unified flavour models, Phys. Rev. D 84 (2011) 117301 [arXiv:1107.3728] [inSPIRE].

[33] D. Marzocca, S.T. Petcov, A. Romanino and M. Spinrath, Sizeable $\theta_{13}$ from the charged lepton sector in $\mathrm{SU}(5)$, (tri-)bimaximal neutrino mixing and Dirac CP-violation, JHEP 11 (2011) 009 [arXiv:1108.0614] [INSPIRE].

[34] P.S. Bhupal Dev, R.N. Mohapatra and M. Severson, Neutrino mixings in $\mathrm{SO}(10)$ with type II seesaw and $\theta_{13}$, Phys. Rev. D 84 (2011) 053005 [arXiv: 1107.2378] [InSPIRE].

[35] P.S. Bhupal Dev, B. Dutta, R.N. Mohapatra and M. Severson, $\theta_{13}$ and proton decay in a minimal $\mathrm{SO}(10) \times S_{4}$ model of flavor, Phys. Rev. D 86 (2012) 035002 [arXiv:1202.4012] [INSPIRE].

[36] M. Bando, S. Kaneko, M. Obara and M. Tanimoto, Can symmetric texture reproduce neutrino bilarge mixing?, Phys. Lett. B 580 (2004) 229 [hep-ph/0309310] [INSPIRE].

[37] M. Bando, S. Kaneko, M. Obara and M. Tanimoto, Symmetric mass matrix with two zeros in SUSY SO(10) GUT, lepton flavor violations and leptogenesis, Prog. Theor. Phys. 112 (2004) 533 [hep-ph/0405071] [inSPIRE].

[38] Particle Data Group collaboration, J. Beringer et al., Review of particle physics, Phys. Rev. D 86 (2012) 010001 [INSPIRE].

[39] C. Jarlskog, Commutator of the quark mass matrices in the standard electroweak model and a measure of maximal CP-violation, Phys. Rev. Lett. 55 (1985) 1039 [INSPIRE].

[40] Z. Maki, M. Nakagawa and S. Sakata, Remarks on the unified model of elementary particles, Prog. Theor. Phys. 28 (1962) 870 [InSPIRE].

[41] B. Pontecorvo, Neutrino experiments and the problem of conservation of leptonic charge, Sov. Phys. JETP 26 (1968) 984 [Zh. Eksp. Teor. Fiz. 53 (1967) 1717] [InSPIRE].

[42] P.F. Harrison, D.H. Perkins and W.G. Scott, Tri-bimaximal mixing and the neutrino oscillation data, Phys. Lett. B 530 (2002) 167 [hep-ph/0202074] [INSPIRE].

[43] P.F. Harrison and W.G. Scott, Symmetries and generalizations of tri-bimaximal neutrino mixing, Phys. Lett. B 535 (2002) 163 [hep-ph/0203209] [INSPIRE].

[44] M.C. Gonzalez-Garcia, M. Maltoni and T. Schwetz, Updated fit to three neutrino mixing: status of leptonic CP-violation, JHEP 11 (2014) 052 [arXiv:1409.5439] [INSPIRE].

[45] D.V. Forero, M. Tortola and J.W.F. Valle, Global status of neutrino oscillation parameters after Neutrino-2012, Phys. Rev. D 86 (2012) 073012 [arXiv: 1205.4018] [INSPIRE].

[46] G.L. Fogli et al., Global analysis of neutrino masses, mixings and phases: entering the era of leptonic CP-violation searches, Phys. Rev. D 86 (2012) 013012 [arXiv:1205.5254] [INSPIRE]. 